\title{
Visualization of Ion Distribution by the Chemical Imaging Sensor
}

Tatsuo Yoshinobu

Professor

Department of Electronic Engineering, Graduate School of Engineering

E-mail:nov@ecei.tohoku.ac.jp

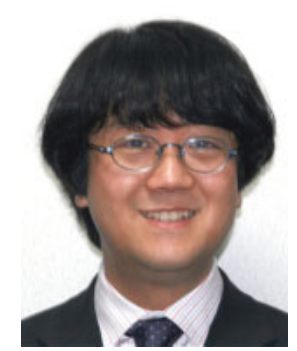

\section{Introduction}

For the visualization of chemical species in the solution, markers and labels such as fluorescent dyes are often utilized. For certain applications in biology, however, toxicity of such dyes may be a problem, especially in the case of cells and tissues in culture. In this study, a label-free method of ion imaging based on a semiconductor device is developed.

Chemical sensors based on semiconductor devices are advantageous for miniaturization, integration with the peripheral circuits and fabrication of various structures on their surfaces with the help of microfabrication techniques such as photolithography. A well-known semiconductor-based chemical sensor is the ion-sensitive field-effect transistor (ISFET) [1,2], which has the field effect structure or the electrolyteinsulator-semiconductor (EIS) structure. The ISFET is put to practical use, for example, in a portable $\mathrm{pH}$ meter. The EIS capacitive sensor [3] and the lightaddressable potentiometric sensor (LAPS) [4] are other examples of chemical sensors based on the same EIS structure. Figure 1 schematically compares the structures of these three semiconductor-based chemical sensors.

Chemical sensors based on the EIS structure detect the change of the distribution of carriers in the semiconductor layer through the field effect, which responds to the change of the ion concentration of the solution in contact with the sensing surface of the insulating layer. The ISFET detects the change of the conductance of the channel between the source and the drain electrodes, whereas the EIS capacitive sensor and the LAPS detect the change of the capacitance of the depletion layer at the semiconductor-insulator interface. In the case of p-type semiconductor, the depletion layer grows thicker and the capacitance of the depletion layer becomes smaller when the sensing surface is more positively charged.

In the LAPS measurement, a photocurrent is generated to detect the change of the capacitance of the depletion layer. The back surface of the sensor plate is illuminated with a light beam modulated at a frequency of several $\mathrm{kHz}$, and the amplitude of the ac photocurrent is measured as a function of the bias voltage applied to the EIS system.
Figure 2(a) shows typical current-voltage characteristics of a pH-sensitive LAPS with a $\mathrm{Ta}_{2} \mathrm{O}_{5}$ film as the insulating layer. A positive bias (applied to the solution with respect to the semiconductor substrate) in Fig. 2(a) corresponds to the depletion and inversion states of the semiconductor layer, and a negative bias corresponds to the accumulation state. In Fig. 2(b), the bias voltage at the inflection point of the current-voltage curve is plotted as a function of the $\mathrm{pH}$ value, showing the $\mathrm{pH}$ sensitivity of $57.9 \mathrm{mV} / \mathrm{pH}$.

The measuring area of a LAPS sensor is restricted to the illuminated area, and therefore, many measuring spots can be defined on the sensing surface of a single sensor plate.

(a)

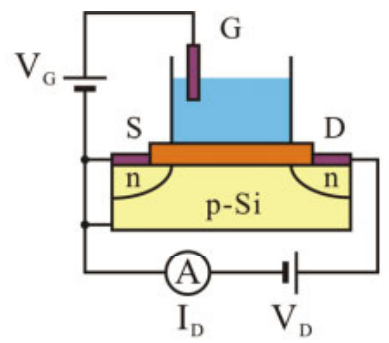

(b)

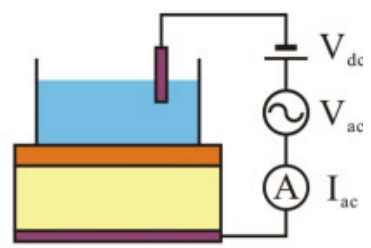

(c)

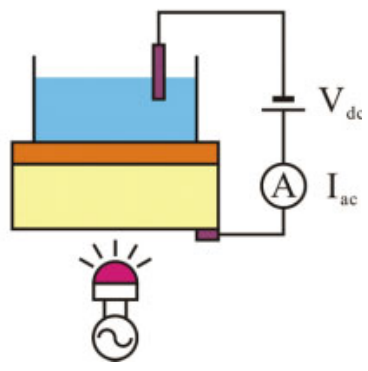

Fig. 1. Structures of (a) ISFET, (b) EIS capacitive sensor and (c) LAPS. 
(a)

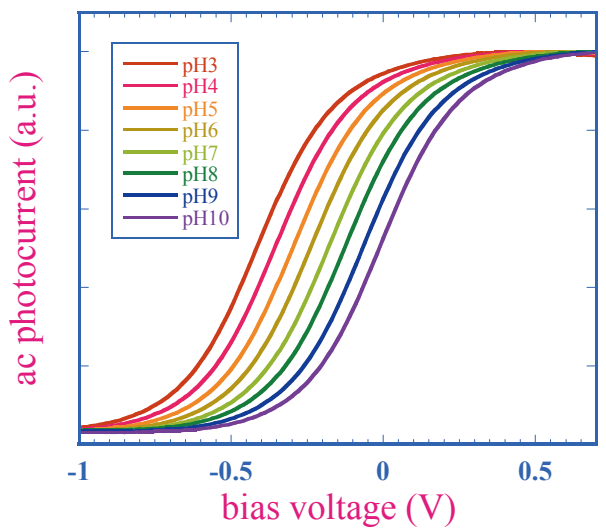

(b)

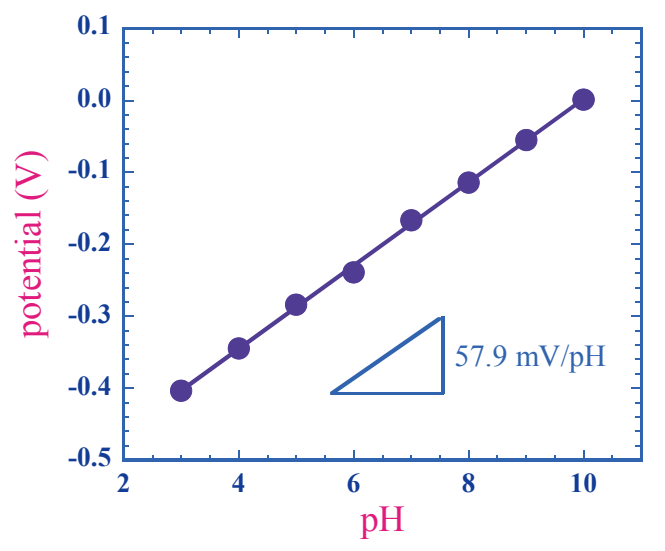

Fig. 2. (a) Current-voltage characteristics and (b) $\mathrm{pH}$ sensitivity of LAPS.

Apart from the $\mathrm{pH}$ sensing applications of LAPS with insulating layers such as $\mathrm{Si}_{3} \mathrm{~N}_{4}, \mathrm{Al}_{2} \mathrm{O}_{3}$ and $\mathrm{Ta}_{2} \mathrm{O}_{5}$, LAPS can be also applied to selective measurement of various ions and molecules by modifying the sensing surface with respective sensing materials.

A family of ion-selective sensors can be realized by forming a membrane with ionophores on the sensing surface. Figure 3 shows the calibration plots of LAPS with PVC membranes containing ionophores sensitive to alkali and alkaline earth metal ions such as $\mathrm{Li}^{+}, \mathrm{K}^{+}$, $\mathrm{Cs}^{+}, \mathrm{Mg}^{2+}$ and $\mathrm{Ca}^{2+}$, respectively. For these sensors, the detection limit was in the $10^{-6} \mathrm{~mol} / \mathrm{l}$ range and the Nernstian sensitivities of $57-59$ and $26-27$ $\mathrm{mV} /$ decade were obtained for monovalent and divalent ions, respectively.

In addition to alkali and alkaline earth metal ions, a variety of LAPS sensors have been developed for detection of various ions and molecules. Anion sensors (nitrate and sulphate) [5], enzyme-based biosensors (urease and cholinesterase) [6] and cadmium sensor based on chalcogenide glasses [7] are examples of such sensors. Thanks to the light-addressability, these functions can be integrated on a single sensor plate. (a)

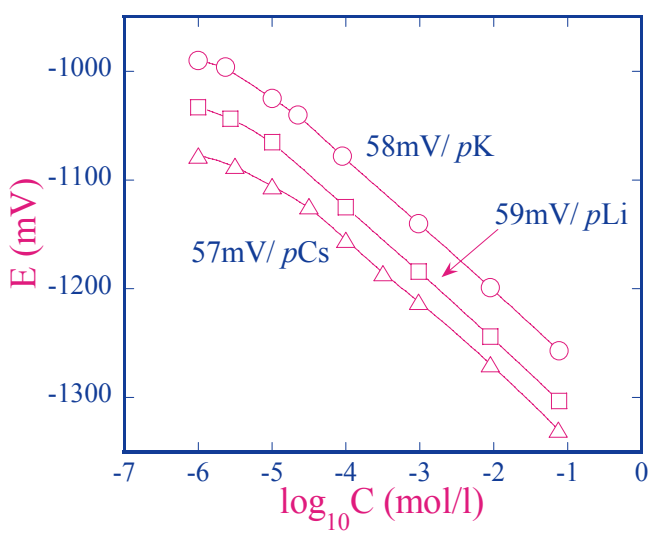

(b)

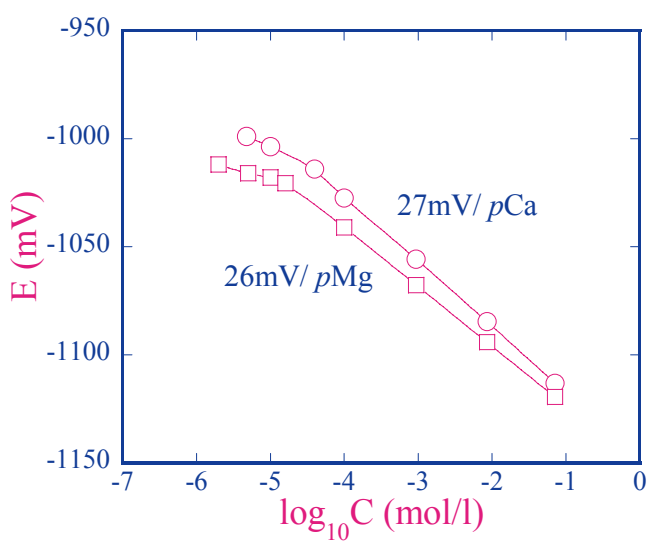

Fig. 3. Calibration plots for (a) monovalent and (b) divalent cations.

\section{Chemical Imaging Sensor 2.1. Principle}

The chemical imaging sensor [8-10] is based on the measurement principle of the LAPS. At the semiconductor-insulator interface of the chemical imaging sensor, the width of the depletion layer is spatially distributed reflecting the spatial distribution of the ion concentration on the sensing surface as shown in Fig.4. In the case of a p-type $\mathrm{Si}$ substrate, for example, the width of the depletion layer is thicker at positions where the cation concentration is higher and the sensing surface is more positively charged, and therefore, the local capacitance of the depletion layer is smaller at these positions.

When the sensor plate is illuminated with a focused laser beam modulated at a certain frequency, an ac photocurrent is generated with an amplitude dependent on the local value of the capacitance of the depletion layer at the illuminated position. Therefore, the photocurrent image obtained by scanning the sensor plate with a focused laser beam gives a map of the ion concentration on the sensing surface. 


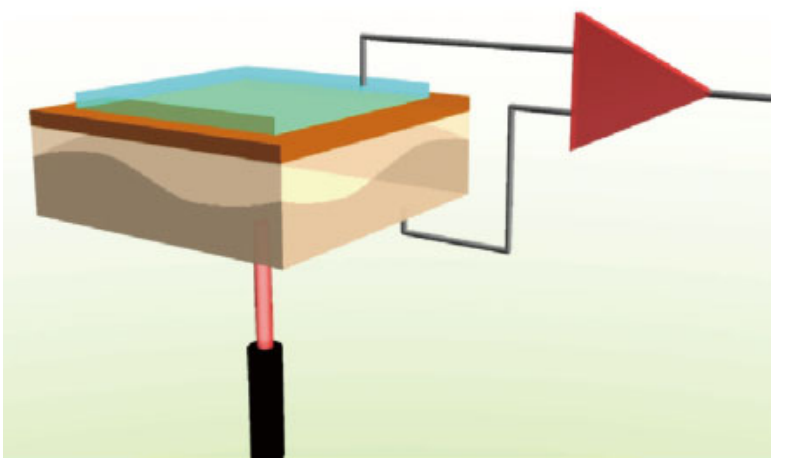

Fig. 4. Principle of the chemical imaging sensor. The photocurrent is dependent on the width of the depletion layer, which is distributed in response to the spatial distribution of the ion concentration on the sensing surface.

One of the advantages of the LAPS-based chemical imaging sensor is the simple structure of the sensor plate. A semiconductor substrate with an insulating layer on the front surface and an ohmic contact on the back surface functions as a chemical imaging sensor without fabricating device structures by photolithography. In addition to the low fabrication cost of a LAPS sensor plate, the flatness of the sensing surface may be an advantage for certain applications, where uniformity is required.

More importantly, in contrast to other devices such as MEA (microelectrode array) or ISFET array, in which the number of pixels and their positions are predetermined by the layout of electrodes or transistors, these parameters can be arbitrarily chosen in the case of LAPS-based chemical imaging sensor, simply by changing the scanning pattern of the light beam. The measured area can be zoomed in, for example, from the size of the silicon wafer down to less than a millimeter.

In analogy to the ion-selective LAPS and enzyme LAPS, the sensing surface of the chemical imaging sensor can be also modified with a variety of ionselective materials and enzymes for visualization of specific ions or molecules. For example, the spatial distribution of urea was visualized with a chemical imaging sensor modified with urease [11].

The spatial resolution of the chemical imaging sensor is determined by various parameters such as the diameter of the focused laser beam, the thickness of the semiconductor layer and the diffusion length of minority carriers in the semiconductor layer. When a combination of silicon and visible light is used, the absorption coefficient in the silicon layer is large and most of the photocarriers are generated in the proximity of the back surface. In this case, the photocarriers diffuse both vertically and horizontally before they arrive at the depletion layer, and therefore, the spatial resolution in this case approximately equals the thickness of the silicon substrate. One of the strategies, therefore, to achieve higher spatial resolution is to make the silicon substrate thinner, which however makes the sensor plate fragile. Another approach is the use of infrared light instead of the visible light. Since the absorption coefficient for an infrared light in silicon is smaller, the light enters deeper into the silicon substrate before generating photocarriers. The photocarriers generated near the depletion layer contribute to the photocurrent signal after travelling smaller distance, which has the same effect as the reduced thickness of the silicon substrate. By combining these two approaches, i.e., a thinner silicon plate and an infrared light beam, a 5- $\mu \mathrm{m}$ lineand-space pattern has been already resolved.

Yet another approach to realize a higher spatial resolution is the use of thin-film semiconductor layer on a transparent glass substrate instead of a bulk semiconductor plate. A spatial resolution up to $1 \mu \mathrm{m}$ is expected by using a-Si thin film $[12,13]$.

\subsection{Measurement system}

Figure 5 shows the schematic diagram of the chemical imaging sensor system. The computer controls the bias voltage, the modulation frequency and the position of the light beam. The bias voltage is applied to the EIS system through a potentiostat, which defines the potential of the semiconductor substrate with respect to the reference electrode. The light source

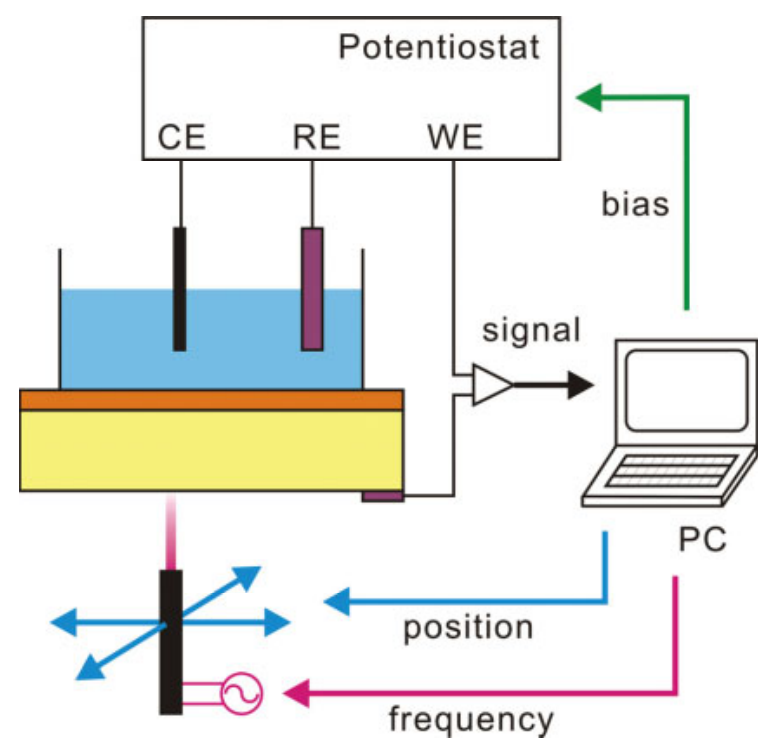

Fig. 5. Schematic diagram of the chemical imaging sensor system. 
is typically a laser diode mounted on a scan stage. A focusing optics is used for high-resolution measurements. The photocurrent signal is amplified and converted into a voltage signal, of which amplitude is recorded.

Figure 6 shows the chemical imaging sensor system based on an optical microscope. The sensor plate and the specimen are mounted on the stage, and the back surface of the sensor plate is illuminated with a focused laser beam from the objective lens mounted on the scanning unit under the stage. The IR camera on top is used for focusing and positioning of the laser beam.

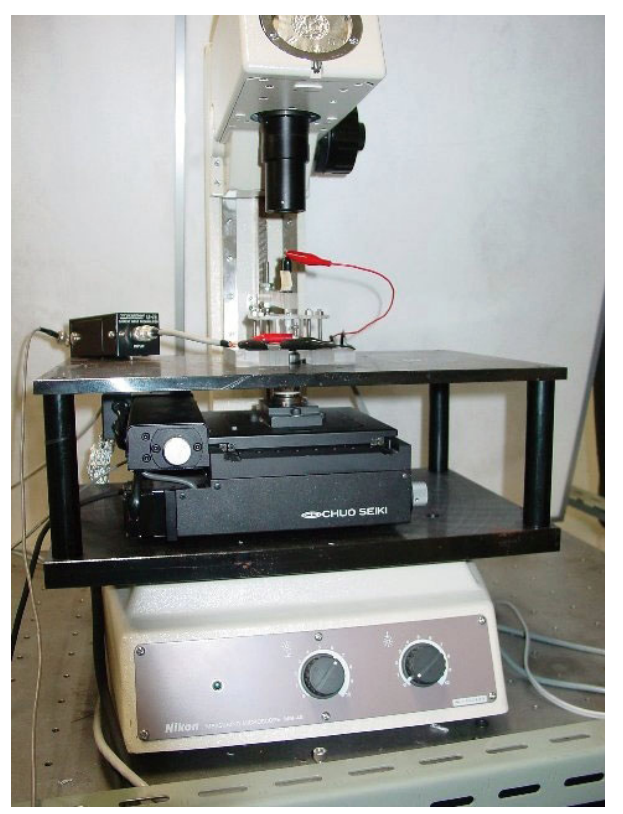

Fig. 6. Scanning system for the chemical imaging sensor.

\subsection{Software}

A control program for this measurement system was developed with LabVIEW (National Instruments). The control program has three measurement modes, i.e., $I-V$ measurement mode, constant-current mode and imaging mode. The program controls the measurement parameters such as the modulation frequency of the laser beam and the bias voltage applied to the EIS system, positions the laser beam, measures the amplitude of the ac photocurrent as the sensor signal and processes the acquired data.

\subsubsection{I-V measurement mode}

The $I-V$ measurement mode of the software is used to obtain the current-voltage curves such as those shown in Fig. 2(a). In this mode, variation of the amplitude of ac photocurrent is recorded as a function of the bias voltage applied to the EIS system. The user interface of the $I-V$ measurement mode is shown in Fig. 7.
Measurement parameters such as the range of bias voltage to be swept and the measuring time per point are set prior to the measurement. After the measurement, the current-voltage curve and its second derivative are graphically displayed on the screen. The voltage shift of the curve, which is the measure of the ion concentration, can be calculated by determining the inflection point of the curve, where the second derivative of the curve equals zero. Using the calibration plot such as the one in Fig. 2(b), the voltage shift of the current-voltage curve along the voltage axis can be converted into the ion concentration of the solution.

The $I-V$ measurement mode is suitable for accurate determination of ion concentration, in case the ion concentration does not rapidly change with time and imaging is not necessary.

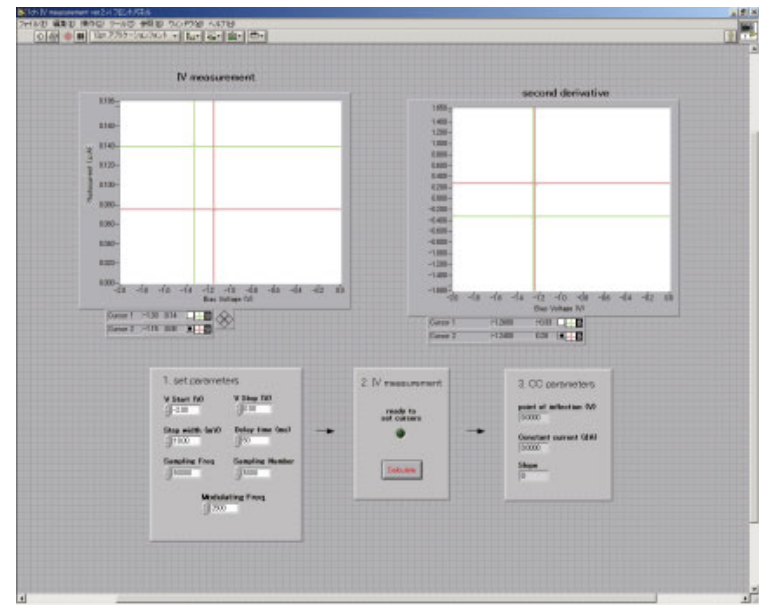

Fig. 7. User interface for the $I-V$ measurement mode, which measures the current-voltage curve of the sensor to determine the ion concentration.

\subsubsection{Constant-current mode}

Whereas the $I-V$ measurement mode sweeps the specified range of bias voltage to obtain currentvoltage curves, the constant-current mode uses feedback control of the bias voltage to maintain the sensor signal, i.e., the amplitude of the ac photocurrent at a constant value [14]. Under this situation, the width of the depletion layer in the semiconductor substrate is kept constant. The bias voltage applied to the EIS system to maintain the constant ac photocurrent is recorded as a function of time as shown in Fig. 8.

The temporal recording of the bias voltage directly corresponds to the shift of the current-voltage curve along the voltage axis, which reflects the temporal change of ion concentration. 


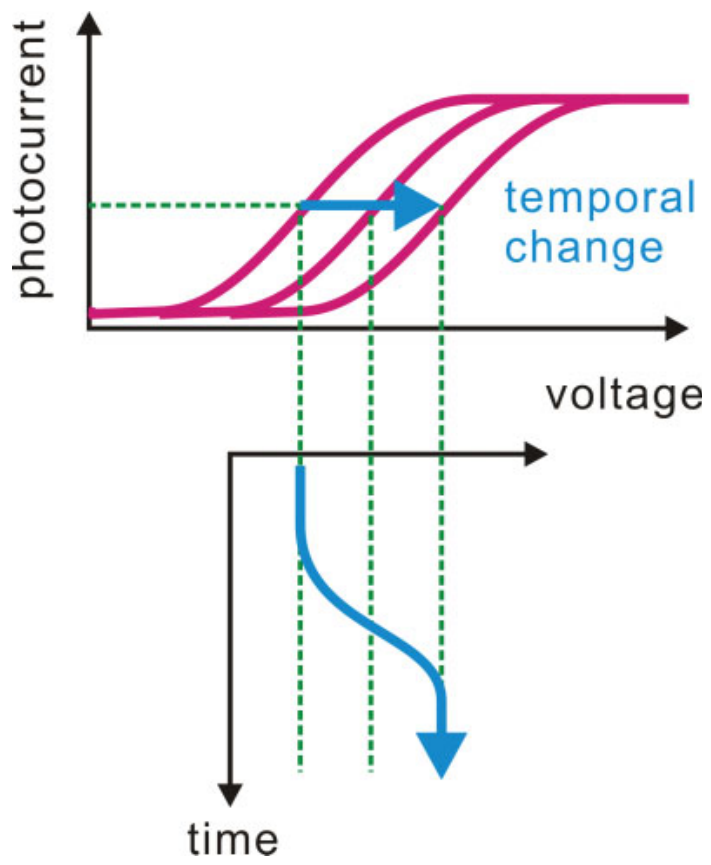

Fig. 8. Temporal recording of the potential change in the constant-current mode. The bias voltage applied to the EIS system is recorded as a function of time, which corresponds to the temporal change of the ion concentration.

This mode can be applied, for example, to the determination of reaction rate, as it can record the ion concentration at the measured position as a function of time. Monitoring of $\mathrm{pH}$ change during an enzymatic reaction is an example of such measurement.

The constant-current mode is also suitable for online monitoring of the ion concentration in a flow-through system, in which the variation of the ion concentration in a stream of solution is recorded as a function of time.

Figure 9(a) shows the user interface of the constantcurrent mode. Prior to the constant-current mode measurement, the current-voltage curve is measured in a specified range of bias voltagee. Using a cursor, the constant current is set near the inflection point of the current-voltage curve, where the slope is maximum. The value of the slope at the set point is used to determine the feedback, by converting the error signal into the voltage shift of the curve.

Fig. 9(b) shows an example of temporal recording of the bias voltage responding to buffer solutions of $\mathrm{pH} 4$ to 10 , which were successively brought into contact with the sensing surface. The bias voltage was changed from $-0.832 \mathrm{~V}$ to $-0.518 \mathrm{~V}$ for the $\mathrm{pH}$ change of 4 to 10 , with an average $\mathrm{pH}$ sensitivity of $52.3 \mathrm{mV} / \mathrm{pH}$. (a)

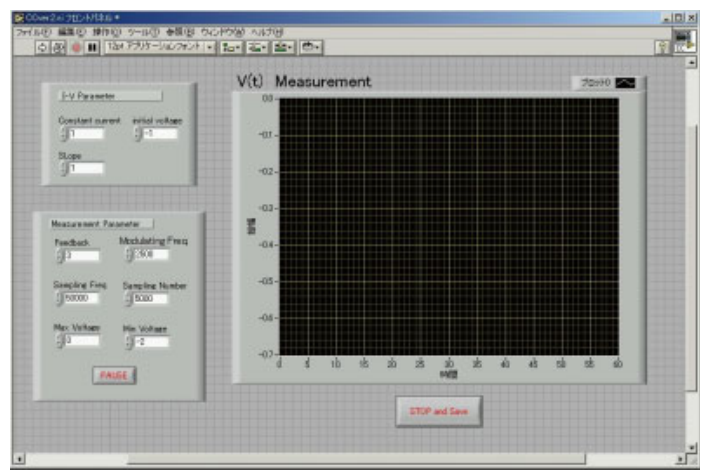

(b)

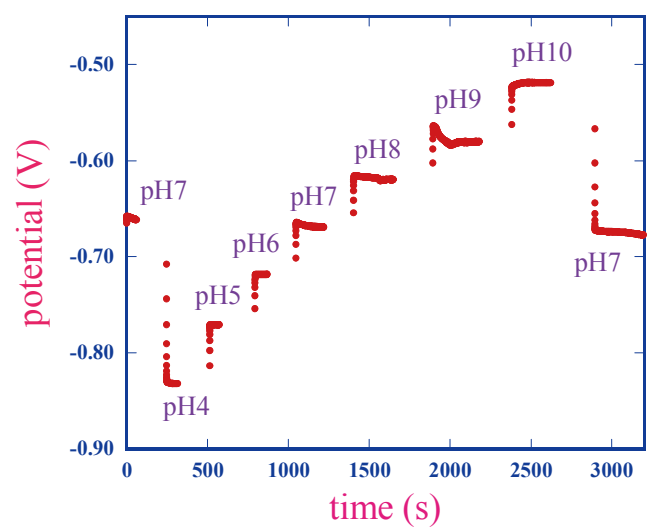

Fig. 9. (a) User interface for the constant-current mode, which measures the potential change as a function of the time. (b) An example of temporal recording of $\mathrm{pH}$ change.

\subsubsection{Imaging mode}

The imaging mode is used to visualize the twodimensional distribution of ion concentration of the solution in contact with the sensing surface. Instead of measuring the current-voltage curve at each pixel, which is too much time-consuming, the bias voltage is fixed at a constant value and the amplitude of the ac photocurrent is recorded at each pixel during a laser scan.

The user interface of the imaging mode is shown in Fig.10. Measurement parameters such as the bias voltage, scan area, resolution (number of pixels), and scan speed are set prior to the measurement.

The measured data can be displayed as a "current image" either in a density map or in a color map. The current image can be converted into a "potential image" using the average slope of the current-voltage curve, and then further converted into an "ion concentration image" using the sensitivity value (voltage shift per decade of ion concentration) of the sensor plate. 


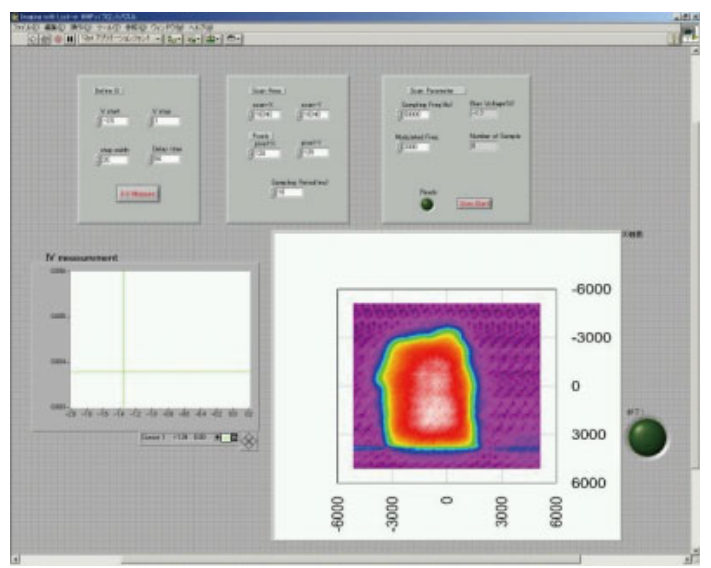

Fig. 10. User interface for the imaging-mode operation. The amplitude of ac photocurrent is measured at each pixel on the sensing surface, and the current image is displayed in a color map.

\subsection{Examples of chemical images}

Possible applications of the chemical imaging sensor include visualization of electrochemical reactions and observation of biological specimens [15-19].

Figure 11 is an example of the visualization of biological specimens. In this example, the surrounding of an E. coli colony cultured on agar is acidified due to the metabolic activity. By using the chemical imaging sensor, biological activities can be measured visually and quantitatively.

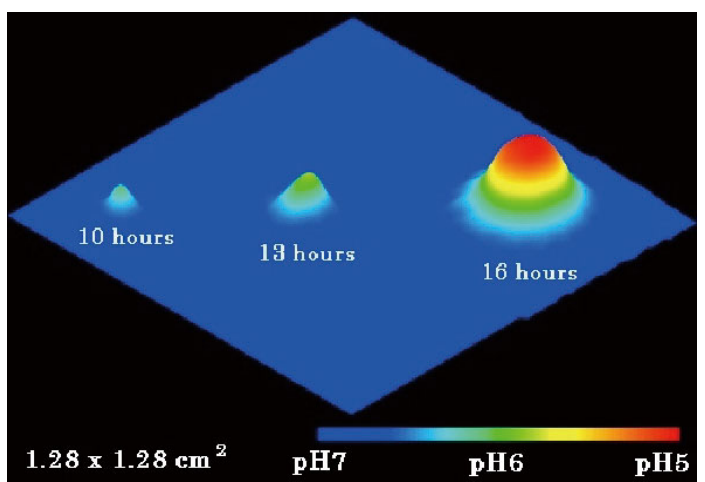

Fig. 11. Visualization of the $\mathrm{pH}$ distribution around an $E$. coli colony after incubation of 10, 13 and 16 hours.

Figure 12 shows an example of visualization of an electrochemical system. In Fig. 12(a), the temporal change of $\mathrm{pH}$ distribution due to diffusion of ions after electrolysis of $0.01 \mathrm{M} \mathrm{NaCl}$ solution is visualized. In this experiment, a pair of Pt electrode separated by 6 $\mathrm{mm}$ was used for electrolysis, and the subsequent change of $\mathrm{pH}$ distribution was recorded by the chemical imaging sensor. (a)

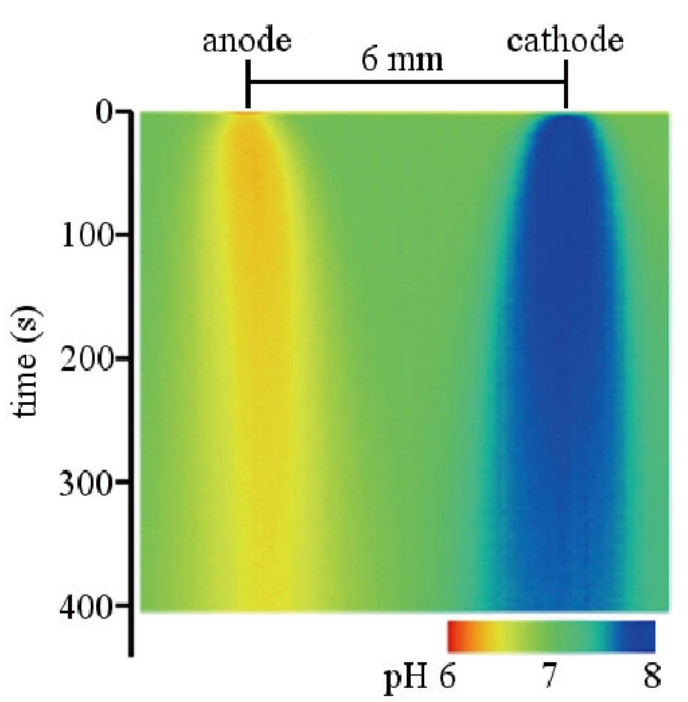

(b)

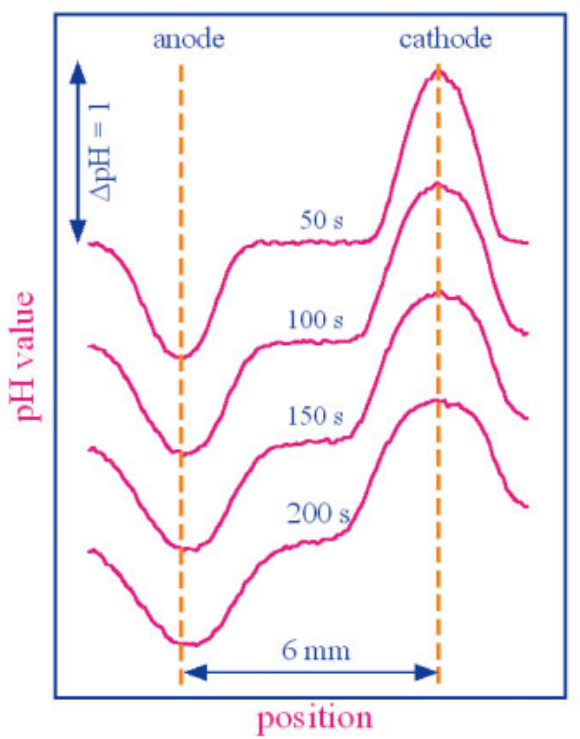

(c)

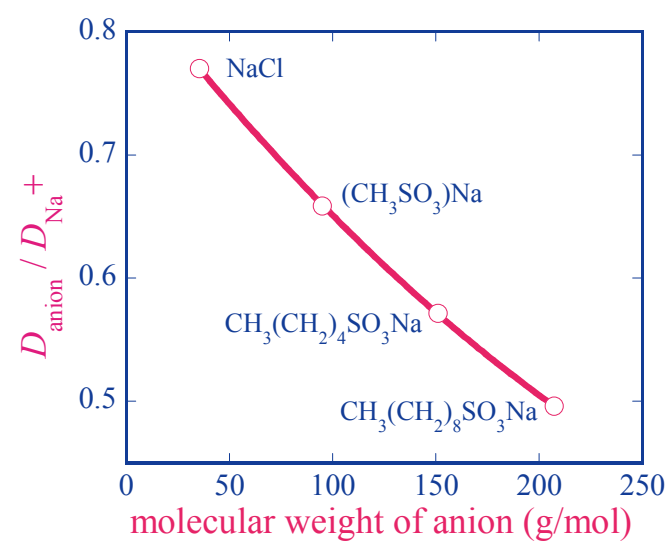

Fig. 12. (a) Redistribution of $\mathrm{pH}$ between two electrodes due to diffusion of ions after electrolysis. (b) $\mathrm{pH}$ profiles at 50,100, 150 and $200 \mathrm{~s}$ after electrolysis. (c) Diffusion coefficients of various anions (normalized to that of $\mathrm{Na}^{+}$) plotted as a function of the molecular weight. 
In Fig. 12(b), the $\mathrm{pH}$ profiles on a line perpendicular to the electrodes at 50,100, 150 and $200 \mathrm{~s}$ after electrolysis are shown. Due to the diffusion of ions, the width of the acidic and basic regions in the proximity of electrodes become broader with time according to the relationship: $W \propto \sqrt{D t}$, where $W$ is the width of the acidic or basic region, $D$ is the diffusion constant, and $t$ is the time after electrolysis. By fitting the diffusion equation to the $\mathrm{pH}$ profiles, the diffusion constants of cations and anions can be determined.

A series of experiments were carried out for electrolytic solutions of $0.01 \mathrm{M} \mathrm{NaCl},\left(\mathrm{CH}_{3} \mathrm{SO}_{3}\right) \mathrm{Na}$, $\mathrm{CH}_{3}\left(\mathrm{CH}_{2}\right)_{4} \mathrm{SO}_{3} \mathrm{Na}$ and $\mathrm{CH}_{3}\left(\mathrm{CH}_{2}\right)_{8} \mathrm{SO}_{3} \mathrm{Na}$, respectively. In Fig. 12(c), the diffusion constants of anions with different molecular weights (normalized to the diffusion constant of $\mathrm{Na}^{+}$) are plotted as a function of the molecular weight. In this way, the chemical imaging sensor enables direct observation and quantitative analysis of chemical reaction and diffusion.

\section{Rapid Scanning of Chemical Images 3.1. Principle}

In the imaging mode, as the amplitude of ac photocurrent is measured one by one for each pixel, the total time required to obtain a chemical image is a big issue.

For example, the total time required for an acquisition of an image at a resolution of $128 \times 128=$ 16,384 pixels is about 163.84 seconds or nearly 3 minutes, assuming that the measurement time per pixel is $10 \mathrm{~ms}$. Due to this rather long measurement time, the chemical imaging sensor has not been applicable to observation of rapidly changing specimens. The measurement time per pixel cannot be smaller than a period of light modulation, which is typically of the order of $\mathrm{kHz}$. As the signal becomes smaller at higher modulation frequencies [20], a drastic reduction of the measurement time is difficult so far as the readout is done pixel-by-pixel.

A method for simultaneous measurement at multiple positions on the LAPS sensing surface has been proposed on the basis of frequency division multiplex [21]. In this method, different positions on the sensing surface are illuminated with light beams modulated at different frequencies. The measured photocurrent in this case is a superposition of ac photocurrent signals with different frequencies at respective positions, which can be separately extracted by Fourier analysis (Fig. 13).

In this study, this principle was applied to rapid scanning of chemical images. A linear array of light sources is used to simultaneously illuminate different positions with different frequencies, and this array scans the sensing area to obtain a two-dimensional image. In this method, pixels on one line are measured at once, and therefore, the total time of measurement can be reduced depending on the multiplicity. A prototype system with a linear array of 16 LEDs has been developed.

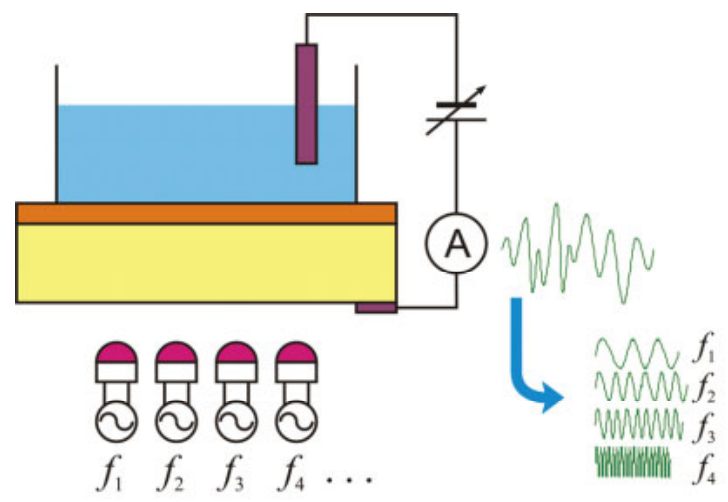

Fig. 13. Simultaneous measurement at different positions on the sensing surface based on frequency division multiplex.

Figure 14 shows the driver circuits for 16 LEDs. The current of each LED is sinusoidally modulated at an independently specified frequency generated with a quartz crystal oscillator and a programmable waveform generator (Analog Devices, AD9833BRM). The number of channels can be increased by adding oscillator modules (small cards in Fig. 14).

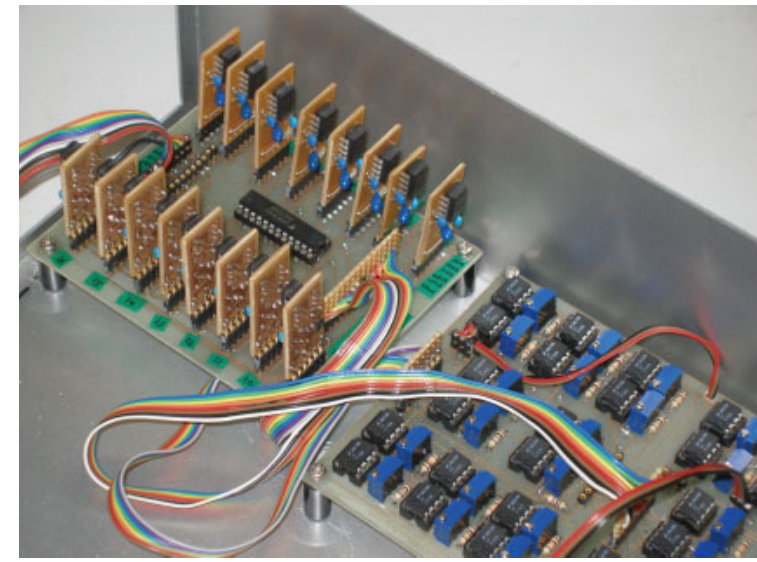

Fig. 14. LED driver unit equipped with programmable function synthesizers. The currents of LEDs are modulated at independently specified frequencies.

Figure 15 shows the FFT spectrum of the photocurrent signal from a LAPS sensor illuminated at 16 different positions with 16 LEDs. The frequency of modulation at each position was set, with a separation of $100 \mathrm{~Hz}$, to be $2.0 \mathrm{kHz}, 2.1 \mathrm{kHz}, \ldots, 3.5 \mathrm{kHz}$ and the sampling frequency was $100 \mathrm{kHz}$. In the frequency domain, signals corresponding to these modulation frequencies are observed. The peak height decreases with the modulation frequency, due to the low-pass filtering effect of carrier diffusion in the semiconductor layer. 
In Fig.16, the amplitude of photocurrent signal at each modulation frequency is plotted as a function of the bias voltage applied to the EIS system. In this way, the current-voltage curves at 16 different positions on the sensing surface are simultaneously obtained, which means that ion concentrations at these positions can be simultaneously determined.

For the application to the imaging-mode operation of LAPS, it is necessary to equalize the signals at different frequencies. Additional circuits and software were developed that automatically adjust the intensities of light beams from 16 LEDs.

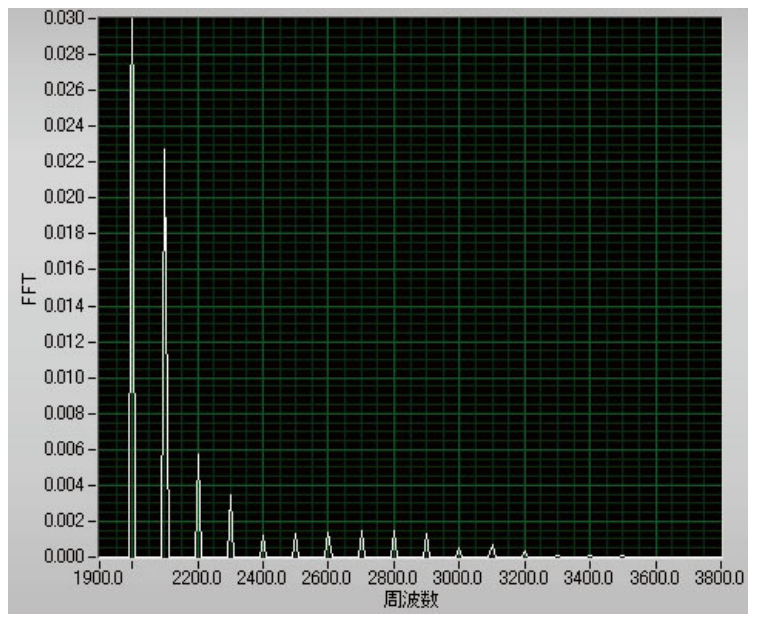

Fig. 15. FFT spectrum of the LAPS signal generated with 16 LEDs with different frequencies, $2.0 \mathrm{kHz}, 2.1$ $\mathrm{kHz}, \cdots, 3.5 \mathrm{kHz}$. The amplitude decreases with the modulation frequency due to the low-pass filtering effect of carrier diffusion.

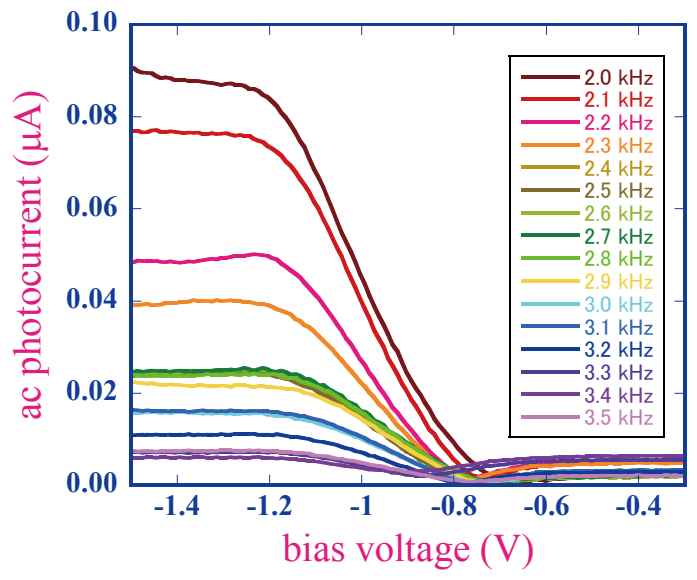

Fig. 16. Simultaneous measurement of the currentvoltage characteristics at 16 points in the sensing area. By calculating the shift of each curve, ion concentration can be determined at 16 different positions.

\subsection{Chemical image scanner}

A prototype of the "chemical image scanner" system was developed (Fig. 17), which has a flatbed structure and scans the LAPS sensor with a linear array of LEDs. The scanner has a linear array of 16 LEDs with a separation of $3.6 \mathrm{~mm}$, mounted on a one-dimensional scan stage, which moves perpendicular to the array. The 16 points on the line are measured simultaneously, and the measurement is repeated 128 times during the one-dimensional scan. The width of the scanned area is $54 \mathrm{~mm}$. A 4-inch LAPS sensor plate is mounted on the stage with a measurement cell and the reference electrode.

With this system, the total time of measurement to obtain a chemical image at a resolution of $16 \times 128$ is 6.4 seconds, when the measurement time per line is set to be $50 \mathrm{~ms}$. In the present case, where the separation of the modulation frequency is $100 \mathrm{~Hz}$, at least $10 \mathrm{~ms}$ of measurement time is required for separation of peaks in the frequency domain.

By increasing the number of LEDs on the array, the resolution can be enhanced without increasing the total time of measurement, which is determined only by the number of pixels in the direction of scan.

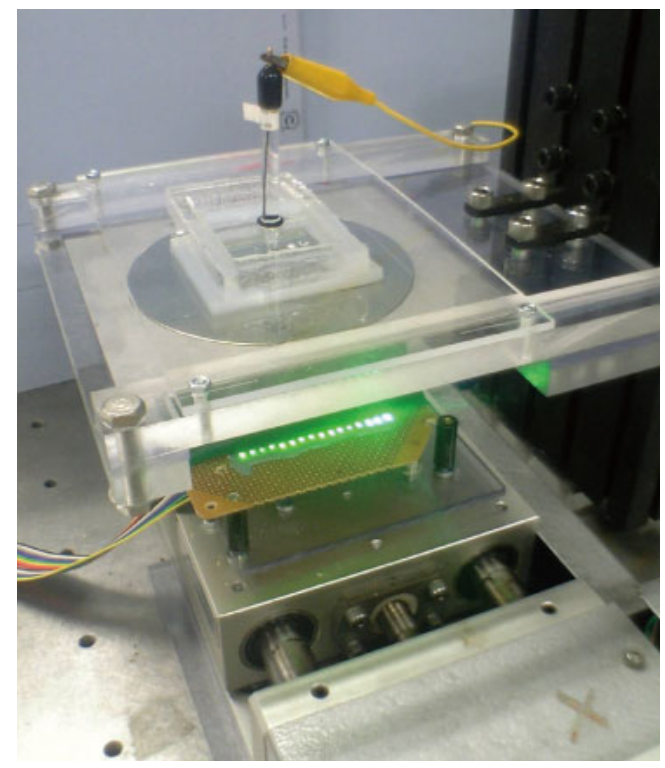

Fig. 17. A prototype of the "chemical image scanner" system with a linear array of 16 LEDs. The size of the sensor is 4 inches in diameter. A chemical image with a resolution of $16 \times 128$ pixels can be obtained in $6.4 \mathrm{~s}$.

Figure 18 shows an example of chemical images of buffer solutions with $\mathrm{pH} 4$ to 10 obtained with this system. The photocurrent increases with the $\mathrm{pH}$ value as shown in Fig. 19, in accordance with the currentvoltage characteristics of a LAPS sensor with a n-type Si substrate. 


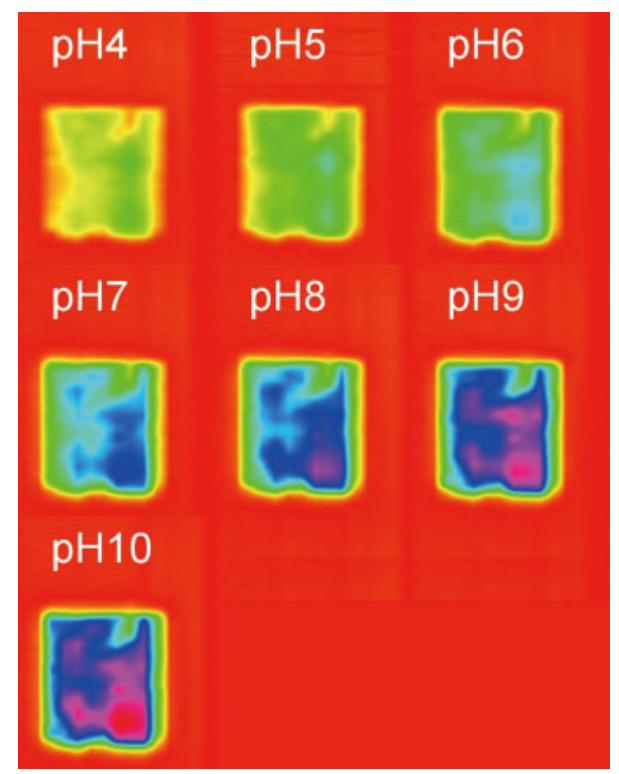

Fig. 18. Current images of $\mathrm{pH}$ buffer solutions measured with the new scan system.

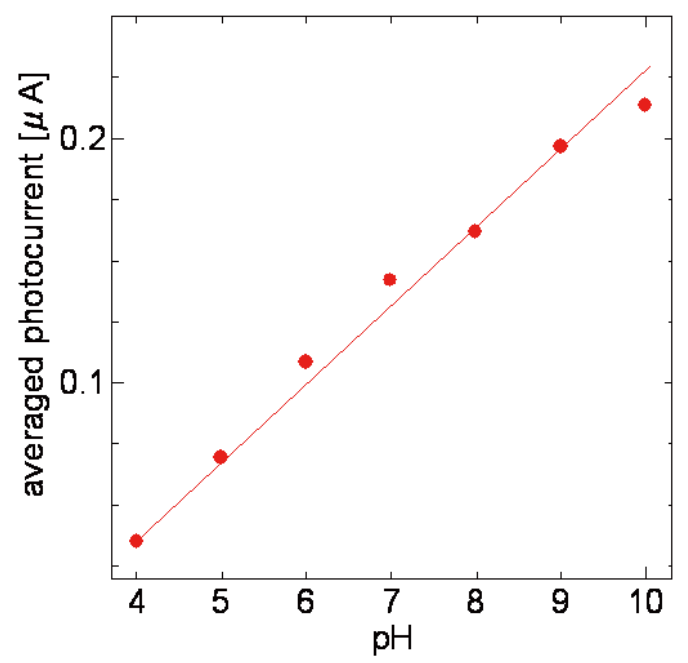

Fig. 19. Dependence of the average value of photocurrent on $\mathrm{pH}$.

\section{Prospects}

In order to develop the applications of the chemical imaging sensor, following aspects of the chemical imaging sensor must be studied.

\section{(1) Performance}

For the precise detection of smaller structures, the spatial resolution and the $\mathrm{S} / \mathrm{N}$ ratio of the chemical imaging sensor must be improved. High-speed measurement is necessary for dynamic measurement.

\section{(2) Multisensor}

By modifying the sensing surface, distributions of various chemical species other than protons $(\mathrm{pH})$ can be also visualized. Multisensors can be built by integrating membranes with sensitivities to different chemical species on a single sensor plate [22-25].

\section{(3) Large-area sensor}

For the measurement of large specimens, large-area sensors and scanners must be developed. For the scanning of a large area, reduction of the measuring time is important, for example, by using more than one light source for the scan.

(4) Application to microfluidic devices

By constructing microchannels on the sensing surface, the distribution of the chemical species inside the channel can be visualized.

\section{Acknowledgement}

The author acknowledges the support of the 2007 Global COE Program "Global Nano-Biomedical Engineering Education and Research Network Centre", Tohoku University.

\section{References}

[1] Bergveld P. Development of an ion-sensitive solidstate device for neurophysical measurements. IEEE Trans Biomed Eng BME-17, 70, 1970.

[2] Matsuo T and Wise KD. An integrated field-effect electrode for biopotential recording. IEEE Trans Biomed Eng BME-21, 485-487, 1974.

[3] Schöning MJ, Poghossian A, Yoshinobu T, and Lüth H. Semiconductor-based field-effect structures for chemical sensing. SPIE Proc 4205, 188-198, 2001.

[4] Hafeman DG, Wallace Parce J, and McConnel HM. Light-addressable potentiometric sensor for biochemical systems. Science 240, 1182-1185, 1988.

[5] Mourzina YG, Ermolenko YE, Yoshinobu T, Vlasov YG, Iwasaki $\mathrm{H}$, and Schöning MJ. Anionselective light-addressable potentiometric sensors (LAPS) for the determination of nitrate and sulphate ions. Sensors and Actuators B 91, 32-38, 2003.

[6] Mourzina YG, Yoshinobu T, Ermolenko YE, Vlasov YG, Schöning MJ, and Iwasaki $H$. Immobilization of urease and cholinesterase on the surface of semiconductor transducer for the development of light-addressable potentiometric sensors. Microchim. Acta 144, 41-50, 2004.

[7] Kloock JP, Moreno L, Bratov A, Huachupoma S, Xu J, Wagner T, Yoshinobu T, Ermolenko YE, Vlasov YG, and Schöning MJ. PLD-prepared cadmium sensors based on chalcogenide glasses - ISFET, LAPS and $\mu$ ISE semiconductor structures. Sensors and Actuators B 118, 149-155, 2006.

[8] Nakao M, Yoshinobu T, and Iwasaki H. Scanninglaser-beam semiconductor $\mathrm{pH}$-imaging sensor. Sensors and Actuators B 20, 119-123, 1994.

[9] Nakao M, Yoshinobu $T$, and Iwasaki $H$. Improvement of spatial resolution of a laser-scanning 
pH-imaging sensor. Jpn J Appl Phys 33, L394-L397, 1994.

[10] Yoshinobu T, Iwasaki H, Ui Y, Furuichi K, Ermolenko $\mathrm{Yu}$, Mourzina $\mathrm{Yu}$, Wagner T, Näther N, and Schöning MJ. The light-addressable potentiometric sensor for multi-ion sensing and imaging. Methods 37, 94-102, 2005.

[11] Inoue S, Yoshinobu T, and Iwasaki H. Chemical imaging sensor using enzyme. Sensors and Actuators $B$ 32, 23-26, 1996.

[12] Yoshinobu T, Schöning MJ, Finger F, Moritz M, and Iwasaki H. Fabrication of thin-film LAPS with amorphous silicon. Sensors 4, 163-169, 2004.

[13] Yoshinobu T, Moritz W, Finger W, and Schöning MJ. Application of thin-film amorphous silicon to chemical imaging. Mat Res Soc Symp Proc 910, 0910A20-01, 2006.

[14] Yoshinobu T, Ecken H, Poghossian A, Simonis A, Iwasaki H, Lüth H, and Schöning MJ. Constantcurrent-mode LAPS (CLAPS) for the detection of penicillin. Electroanalysis 13, 733-736, 2001.

[15] Nakao M, Inoue S, Oishi R, Yoshinobu $\mathrm{T}$, and Iwasaki $\mathrm{H}$. Observation of microorganism colonies using a scanning-laser-beam $\mathrm{pH}$-sensing microscope. Journal of Fermentation and Bioengineering 79, 163166, 1995.

[16] Yoshinobu T, Oba N, Iwasaki H. Visualization of chemical waves in Belousov-Zhabotinsky reaction by chemical imaging sensors. J Electrochem Soc 144, 3919-3921, 1997.

[17] Yoshinobu T, Iwasaki H, Nakao M, Nomura S, Nakanishi T, Takamatsu S, and Tomita K. Application of chemical imaging sensor to electrogenerated $\mathrm{pH}$ distribution. Jpn J Appl Phys 37, L353-L355, 1998.

[18] Yoshinobu T, Harada $T$, and Iwasaki $H$. Application of the $\mathrm{pH}$-imaging sensor to determining the diffusion coefficients of ions in electrolytic solutions. Jpn J Appl Phys 39, L318-L420, 2000.

[19] Yoshinobu T, Ecken H, Ismail ABM, Iwasaki H, Lüth $H$, and Schöning MJ. Chemical imaging sensor and its application to biological systems. Electrochimica Acta 47, 259-263, 2001.

[20] Satore M, Adami M, Nicolini C, Bousse L, Mostarshed S, and Hafeman D. Minority carrier diffusion length effects on light-addressable potentiometric sensor (LAPS) devices. Sensors and Actuators A 32, 431-436, 1992.

[21]Zhang QT, Wang P, Parak WJ, George M, and Zhang GY. A novel design of multi-light LAPS based on digital compensation of frequency domain. Sensors and Actuators B 73, 152-156, 2001.

[22] Schöning MJ, Wagner T, Wang C, Otto R, and Yoshinobu T. Development of a handheld 16 channel pen-type LAPS for electrochemical sensing. Sensors and Actuators B 108, 808-814, 2005.

[23] Wagner T, Yoshinobu T, Rao CR, Otto R, and Schöning MJ. All-in-one solid-state device based on a light-addressable potentiometric sensor platform. Sensors and Actuators B 117, 472-479, 2006.
[24] Wagner T, Rao C, Kloock JP, Yoshinobu T, Otto R, and Schöning MJ. LAPS card - a novel chip cardbased light-addressable potentiometric sensor (LAPS). Sensors and Actuators B 118, 33-40, 2006.

[25] Wagner T, Molina R, Yoshinobu T, Kloock JP, Biselli M, Canzoneri M, Schnitzler T, and Schöning MJ. Handheld multi-channel LAPS device as a transducer platform for possible biological and chemical multi-sensor applications. Electrochimica Acta 53, 305-311, 2007. 